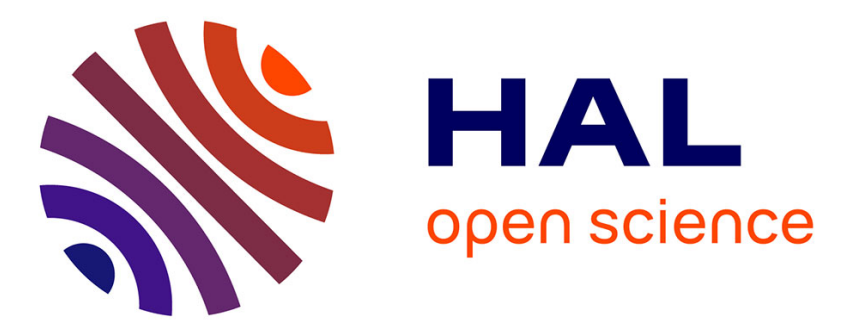

\title{
Periodic homogenization for Kelvin-Voigt viscoelastic media with a Kelvin-Voigt viscoelastic interphase
}

Loïc Daridon, Christian Licht, Somsak Orankitjaroen, Stéphane Pagano

\section{To cite this version:}

Loïc Daridon, Christian Licht, Somsak Orankitjaroen, Stéphane Pagano. Periodic homogenization for Kelvin-Voigt viscoelastic media with a Kelvin-Voigt viscoelastic interphase. European Journal of Mechanics - A/Solids, 2016, 58, pp.163-171. 10.1016/j.euromechsol.2015.12.007 . hal-01282112

\section{HAL Id: hal-01282112 \\ https://hal.science/hal-01282112}

Submitted on 3 Mar 2016

HAL is a multi-disciplinary open access archive for the deposit and dissemination of scientific research documents, whether they are published or not. The documents may come from teaching and research institutions in France or abroad, or from public or private research centers.
L'archive ouverte pluridisciplinaire HAL, est destinée au dépôt et à la diffusion de documents scientifiques de niveau recherche, publiés ou non, émanant des établissements d'enseignement et de recherche français ou étrangers, des laboratoires publics ou privés. 


\title{
Periodic homogenization for Kelvin-Voigt viscoelastic media with a Kelvin-Voigt viscoelastic interphase
}

\author{
Loic DARIDON ${ }^{\mathrm{a}, \mathrm{c}, \star}$, Christian LICHT $^{\mathrm{a}, \mathrm{b}}$, \\ Somsak ORANKITJAROEN ${ }^{\mathrm{b}}$, Stéphane PAGANO ${ }^{\mathrm{a}, \mathrm{c}}$ \\ ${ }^{\text {a }}$ Laboratoire de Mécanique et Génie Civil (LMGC), Université de Montpellier, \\ CNRS, Montpellier, France \\ ${ }^{\mathrm{b}}$ Department of Mathematics, Faculty of Science, Mahidol University, Bangkok, \\ Thailand \\ ${ }^{\mathrm{c}}$ Laboratoire de Micromécanique et d'Intégrité des Structures, Université de \\ Montpellier, CNRS, IRSN, Montpellier, France
}

\begin{abstract}
We studied the effective behavior of a composite made of a periodic distribution of inclusions linked to a matrix by a very thin layer, with the three components being of linear Kelvin-Voigt viscoelastic type. The effective behavior, derived by a rigorous mathematical homogenization method, is not of Kelvin-Voigt type but instead involves an additional fading memory term. The influence of various parameters of the composite were numerically analyzed through a finite elements method.
\end{abstract}

Key words: Kelvin-Voigt viscoelasticity, Viscoelastic interface, Homogenization; 


\section{Introduction}

Composite mechanical behavior clearly depends on the nature of the constituents but also on that of the interfaces between them [1,2]. Making perfectly bonded inclusions in a matrix during composite materials manufacturing is still a major technological issue [3]. Moreover, estimating the effective behavior of viscoelastic composites is of growing interest due to their intensive use in many engineering components under dynamic loadings, particularly for sandwich structures $[4,5]$ but also for granular composites [6]. The nature of the matrix/reinforcement interface can evolve from perfect bonding to complete debonding [7]. This pattern can result from the loading or coupling agent (Figure 1 and Figure 2). Dynamic loadings on this type of material clearly induce microstructural damage, such as matrix-inclusion debonding [8] and [9].

Around 30 years ago, $[10,11,12]$ established that the effective behavior of a heterogeneous solid with perfectly bonded linear Kelvin-Voigt viscoelastic components is no longer of Kelvin-Voigt type but instead has a long, but fading, memory. Here we consider a composite made of a periodic distribution of inclusions linked to a matrix by very thin layers. The three phases are assumed to be of Kelvin-Voigt type but the coefficients of elasticity and viscosity of the layers are lower than those of the matrix and inclusions $[13,14,15,16]$. Recently, [17] proposed a semi-analytical incremental model using the Eshelby solution

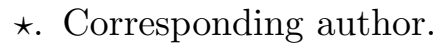

Email addresses: loic.daridon@umontpellier.fr (Loic DARIDON ), christian.licht@umontpellier.fr (Christian LICHT), somsak.ora@mahidol.ac.th (Somsak ORANKITJAROEN), stephane.pagano@umontpellier.fr (Stéphane PAGANO). 
for two elastic phases associated with a non-linear traction-separation law at the interface. Other studies have also highlighted an influence of the viscoelastic mechanical properties of the interface on the damping of composite plates $[18,19]$

In this paper, we propose a theoretical and numerical approach where the three components (inclusions, matrix, interface) are viscoelastic. First, by homogenization we derive the effective behavior which is also not of Kelvin-Voigt type but instead involves an additional memory term with exponential decay. We then use a finite element method to quantify the influence of various geometrical and mechanical parameters on the kernel associated with the fading memory term.

\section{Effective behavior}

The heterogeneity of a three-phase viscoelastic body $\mathbb{S}$ occupying a domain $\Omega$ with a smooth boundary $\partial \Omega$ can be described as follows. Let $Y=(0,1)^{3}$ be the unit cell of $\mathbb{R}^{3}$ and $I$ be a domain strongly included in $Y$ with a smooth boundary $S$. Let $h$ be a small real number in $(0, \operatorname{dist}(S, \partial Y))$ with

the sets $B_{h}:=\{y \in Y ; \operatorname{dist}(y, S)<h\}, I_{h}:=\left\{y \in I ; y \notin \bar{B}_{h}\right\}, Y_{h}^{*}:=$ $Y \backslash\left(B_{h} \cup \bar{I}_{h}\right)$. For clarity, we propose a 2D representation of different regions of the spaces introduced above in Figure 3. Let $\varepsilon$ be another small real number with $J_{\varepsilon}:=\left\{j \in \mathbb{Z}^{3} ; \varepsilon(j+Y) \subset \Omega\right\}$ then $I_{h \varepsilon}:=\bigcup_{j \in J_{\varepsilon}} \varepsilon\left(j+I_{h}\right), B_{h \varepsilon}:=$ $\bigcup_{j \in J_{\varepsilon}} \varepsilon\left(j+B_{h}\right)$ and $\Omega_{h \varepsilon}^{*}:=\Omega \backslash\left(\bar{I}_{h \varepsilon} \cup B_{h \varepsilon}\right)$ standing for the geometric sets occupied by the periodic distribution of the inclusions, the thin interphase, and the matrix, respectively. Hereafter, the subscript letters $e$ and $v$ refer to elastic and viscoelastic mechanical characteristics, respectively. The materials 
constituting $\mathbb{S}$ are assumed to be linearly viscoelastic of Kelvin-Voigt type with $A^{s^{\prime}}(y)$ and $B^{s^{\prime}}(y)$ denoting the elasticity and viscosity tensors. More precisely, we assume that there are four mappings $A_{M}, B_{M}, A_{I}, B_{I}$ defined on $Y$ and taking values in the space of $4^{\text {th }}$ order tensors, with four real positive numbers $\lambda_{e}, \mu_{e}, \lambda_{v}, \mu_{v}$, such that:

$$
\begin{aligned}
& A^{s^{\prime}}(y)= \begin{cases}A_{M}(y) & \text { if } y \in Y_{h}^{*} \\
\lambda_{e} \theta+2 \mu_{e} I d & \text { if } y \in B_{h} \\
A_{I}(y) & \text { if } y \in I_{h}\end{cases} \\
& B^{s^{\prime}}(y)= \begin{cases}B_{M}(y) & \text { if } y \in Y_{h}^{*} \\
\lambda_{v} \theta+2 \mu_{v} I d & \text { if } y \in B_{h} \\
B_{I}(y) & \text { if } y \in I_{h}\end{cases}
\end{aligned}
$$

with $s^{\prime}=\left(h, \lambda_{e}, \mu_{e}, \lambda_{v}, \mu_{v}\right)$ and $\theta$ being the mapping defined on the space $S^{3}$ of $3 \times 3$ symmetric matrices by :

$$
e \in S^{3} \mapsto \theta e=(\operatorname{tr} e) I d \in S^{3}
$$

where $I d$ is the identity matrix of $S^{3}$ and $\operatorname{tr} e$ is the trace of $e$. If the same symbols denote the extensions into $\mathbb{R}^{3}$ by $Y$-periodicity of these two functions $A^{s^{\prime}}$ and $B^{s^{\prime}}$, then the elasticity and viscosity tensors which involve the sextuple of parameters $s:=\left(\varepsilon, s^{\prime}\right)$, read as :

$$
A^{s}(x)=A^{s^{\prime}}(x / \varepsilon), \quad B^{s}(x)=B^{s^{\prime}}(x / \varepsilon) \quad \forall x \in \Omega \text {. }
$$


Hence, the quasi-static evolution of $\mathbb{S}$ clamped on $\partial \Omega$ subjected to body forces of density $f$ is governed by :

$$
\left(P_{s}\right): \begin{cases}\operatorname{div} \sigma^{s}+f=0 & \text { in } \Omega \\ \sigma^{s}=A^{s} e\left(u^{s}\right)+B^{s} e\left(\dot{u}^{s}\right) & \text { in } \Omega \\ u^{s}=0 & \text { on } \partial \Omega \\ u^{s}(\cdot, 0)=u_{0}^{s} & \text { in } \Omega\end{cases}
$$

where $u^{s}, e\left(u^{s}\right), \sigma^{s}$ stand for displacement, strain and stress fields, while the upper dot denotes the time derivative and $u_{0}^{s}$ the initial state. If $f$ is smooth enough, problem $\left(P_{s}\right)$ may be formulated in terms of an evolution equation in a Hilbert space of possible states with finite strain energy [11,12]. Then the theory of semi-groups of linear operators implies the existence and uniqueness of a weak solution of $\left(P_{s}\right)$. Therefore, the effective behavior of the strongly heterogeneous body may be derived by a study of the asymptotic behavior of $\left(u^{s}, \sigma^{s}\right)$ when $s$ tends to 0 with the condition :

$$
\exists\left(\bar{\lambda}_{e}, \bar{\mu}_{e}, \bar{\lambda}_{v}, \bar{\mu}_{v}\right) \in[0, \infty]^{4} \text { s. t. }\left(\bar{\lambda}_{e}, \bar{\mu}_{e}, \bar{\lambda}_{v}, \bar{\mu}_{v}\right)=\lim _{s \rightarrow 0} \frac{1}{2 h}\left(\lambda_{e}, \mu_{e}, \lambda_{v}, \mu_{v}\right)
$$

Because such a study has been already done from a rigorous mathematical point of view in [20] and that one of the goals of this present paper is the study of the influence of some geometric and mechanic parameters, in this section, we confine to heuristically suggest what could be the effective behavior. This is done in three steps. First a suitable assumption 6 on the initial state and a Laplace transform with respect to the time reduce the problem to two problems of periodic homogenization in elastostatics. These two problems which involve additional parameters to the periodicity one, are solved 
by adding arguments from the mathematical modeling of bonding. Finally, as Laplace transform is one to one, this supplies the effective behavior. More precisely, it is convenient $[11,12]$ to assume that "the initial state is elastic", i.e. there exists $g_{0}$ independent of $s$ such that

$$
\begin{cases}\operatorname{div} \sigma_{0}^{s}+g_{0}=0 & \text { in } \Omega \\ \sigma_{0}^{s}=A^{s} e\left(u_{0}^{s}\right) & \text { in } \Omega \\ u_{0}^{s}=0 & \text { on } \partial \Omega .\end{cases}
$$

Hence, if $\hat{z}$ denotes the Laplace transform with respect to the time of a function $z:$

$$
\hat{z}(p)=\int_{0}^{\infty} e^{-p t} z(t) d t
$$

the field $\theta^{s}:=p \widehat{u^{s}}-u_{0}^{s}$ does solve an abstract heterogeneous elastostatic problem :

$$
\begin{cases}\operatorname{div} \xi^{s}-\frac{1}{p} g_{0}=0 & \text { in } \Omega \\ \xi^{s}=C^{s} e\left(\theta^{s}\right) & \text { in } \Omega \\ \theta^{s}=0 & \text { on } \partial \Omega\end{cases}
$$

where

$$
C^{s}(p):=\frac{1}{p} A^{s}+B^{s}
$$

Indeed (6) and (8) are not exactly standard problems of periodic homogenization for elastic bodies because, due to the presence of parameter $s^{\prime}$ in the expression of $A^{s^{\prime}}$ and $B^{s^{\prime}}$, the coefficients of 'elasticity' $A^{s}(x)$ and $C^{s}(p)(x)$ are not of the type $R(x / \varepsilon)$ with $R$ being a fixed function. Nevertheless, by combining arguments of homogenization theory [10] and mathematical modeling 
of soft adhesive bonded joints [21], it is possible to determine the asymptotic behaviors of $\left(u_{0}^{s}, \sigma_{0}^{s}\right)$ and $\left(\theta^{s}, \xi^{s}\right)$. Roughly speaking, if $s^{\prime}$ is kept constant, when $\varepsilon$ tends to zero, the asymptotic behavior of the two previous couples of fields should be of standard homogeneous type with elasticity coefficients given through the solutions to the cell problems :

$$
\begin{aligned}
& \left(\tilde{P}^{e, k l}\right):\left\{\begin{array}{l}
\operatorname{div} \tilde{\sigma}^{e, k l}=0 \quad \text { in } Y \\
\tilde{\sigma}^{e, k l}=A^{s^{\prime}}(y)\left(E^{k l}+e\left(\tilde{u}^{e, k l}\right)\right) \\
\tilde{u}^{e, k l} Y \text {-periodic, } \quad \tilde{\sigma}^{e, k l} n Y \text {-antiperiodic }
\end{array}\right. \\
& \left(\tilde{P}^{p, k l}\right):\left\{\begin{array}{l}
\operatorname{div} \tilde{\sigma}^{p, k l}=0 \quad \text { in } Y \\
\tilde{\sigma}^{p, k l}=\left(\frac{1}{p} A^{s^{\prime}}(y)+B^{s^{\prime}}(y)\right)\left(E^{k l}+e\left(\tilde{u}^{p, k l}\right)\right) \\
\tilde{u}^{p, k l} Y \text {-periodic, } \quad \tilde{\sigma}^{p, k l} n Y \text {-antiperiodic }
\end{array}\right.
\end{aligned}
$$

by

$$
\begin{aligned}
\tilde{A}_{i j k l}^{s^{\prime}} & =\frac{1}{|Y|} \int_{Y}\left(\tilde{\sigma}^{e, k l}\right)_{i j} \mathrm{~d} y \\
\tilde{C}_{i j k l}^{s^{\prime}}(p) & =\frac{1}{|Y|} \int_{Y}\left(\tilde{\sigma}^{p, k l}\right)_{i j} \mathrm{~d} y
\end{aligned}
$$

and $E^{k l}$ is an element of the canonical basis of $S^{3}$, with $n$ being the unit outward normal along $\partial Y$.

Then, according to [21], letting $s^{\prime}$ tend to zero with due account of (5) generates the following candidate for effective coefficients

$$
\begin{aligned}
A_{i j k l}^{\mathrm{eff}} & =\frac{1}{|Y|} \int_{Y}\left(\sigma^{e, k l}\right)_{i j} \mathrm{~d} y \\
C_{i j k l}^{\mathrm{eff}}(p) & =\frac{1}{|Y|} \int_{Y}\left(\sigma^{p, k l}\right)_{i j} \mathrm{~d} y
\end{aligned}
$$


through solutions to the cell problems:

$$
\left(P^{e, k l}\right): \begin{cases}\operatorname{div} \sigma^{e, k l}=0 & \text { in } Y \\ \sigma^{e, k l}=A_{M}\left(E^{k l}+e\left(u^{e, k l}\right)\right) & \text { in } Y \backslash \bar{I} \\ \sigma^{e, k l} \nu=\left(\bar{\lambda}_{e}\left[u^{e, k l}\right]_{\nu}+2 \bar{\mu}_{e}\left[u^{e, k l}\right] \otimes_{S} \nu\right) \nu & \text { on } S \\ \sigma^{e, k l}=A_{I}\left(E^{k l}+e\left(u^{e, k l}\right)\right) & \text { in } I \\ u^{e, k l} Y \text {-periodic, } \quad \sigma^{e, k l} n Y \text {-antiperiodic } & \end{cases}
$$

and $\left(P^{p, k l}\right)$ where $A_{M}, A_{I}, \bar{\lambda}_{e}, \bar{\mu}_{e}$ are respectively replaced by $C_{M, p}:=\frac{1}{p} A_{M}+$ $B_{M}, C_{I, p}:=\frac{1}{p} A_{I}+B_{I}, \bar{\lambda}_{p}:=\frac{1}{p} \bar{\lambda}_{e}+\bar{\lambda}_{v}, \bar{\mu}_{p}:=\frac{1}{p} \bar{\mu}_{e}+\bar{\mu}_{v}$; with $\nu$ being a unit normal along $S$ while $[v]$ denotes the jump of the vector field $v$ across $S$, $[v]_{\nu}=[v] \cdot \nu$ denotes the jump in the direction of $\nu, \otimes_{S}$ is the symmetric tensor product. The solution to problem $\left(P^{v, k l}\right)$, where $A_{M}, A_{I}, \bar{\lambda}_{e}, \bar{\mu}_{e}$ are replaced by $B_{M}, B_{I}, \bar{\lambda}_{v}, \bar{\mu}_{v}$, is respectively denoted by $\left(u^{v, k l}, \sigma^{v, k l}\right)$ and we set $B_{i j k l}^{e f f}=\frac{1}{|Y|} \int_{Y}\left(\sigma^{v, k l}\right)_{i j} \mathrm{~d} y$. Actually, in the cell problems $\left(P^{e, k l}\right)$, the interphase occupying the layer $B_{h}$ is replaced by a mechanical constraint along $S$, the interface the layer shrinks to. The constitutive equations of this constraint depend strongly on the relative behavior of $\lambda_{e}, \mu_{e}, \lambda_{v}, \mu_{v}$ with respect to $h$. If $\bar{\mu}_{e}=+\infty$, there is perfect adhesion between $I$ and $Y \backslash \bar{I}$; if $\bar{\lambda}_{e}=+\infty$, there is bilateral contact between $I$ and $Y \backslash \bar{I}$ with a tangential sliding with or without elastic resistance depending on $\bar{\mu}_{e} \neq 0$ or $\bar{\mu}_{e}=0$; if $\bar{\lambda}_{e}=0$ and $\bar{\mu}_{e}=0, I$ and $Y \backslash \bar{I}$ are free to separate; as soon as one coefficient $\bar{\lambda}_{e}, \bar{\mu}_{e}$ differs from zero or $+\infty$, there is an elastic pull back between $I$ and $Y \backslash \bar{I}$. Similar remarks stand for the cell problems $\left(P^{p, k l}\right)$ with $\bar{\lambda}_{p}, \bar{\mu}_{p}$ in place of $\bar{\lambda}_{e}, \bar{\mu}_{e}$. Thus, it is only for the sake of simplicity that the interphase is assumed isotropic; general elasticity coefficients would involve the limit of their ratio by $2 h$ ! On the other hand, assuming first that $\varepsilon$ is fixed and letting $s^{\prime}$ tend to zero, with $\bar{\mu}_{e} \neq 0$ and 
$\bar{\mu}_{v} \neq 0$, will imply [21] that $\left(u_{0}^{s}, \sigma_{0}^{s}\right)$ and $\left(\theta^{s}, \xi^{s}\right)$ will converge toward $\left(u_{0}^{\varepsilon}, \sigma_{0}^{\varepsilon}\right)$ and $\left(\theta^{\varepsilon}, \xi^{\varepsilon}\right)$ solutions to the following periodic homogenization problems :

$$
\begin{gathered}
\begin{cases}\operatorname{div} \sigma_{0}^{\varepsilon}+g_{0}=0 & \text { in } \Omega \\
\sigma_{0}^{\varepsilon}=A_{M}(x / \varepsilon) e\left(u_{0}^{\varepsilon}\right) & \text { in } M_{\varepsilon} \\
\sigma_{0}^{\varepsilon} \nu_{\varepsilon}=\left(\bar{\lambda}_{e}\left[u_{0}^{\varepsilon}\right]_{\varepsilon} \cdot \nu_{\varepsilon}+2 \bar{\mu}_{e}\left[u_{0}^{\varepsilon}\right]_{\varepsilon} \otimes_{S} \nu_{\varepsilon}\right) \nu_{\varepsilon} & \text { on } S_{\varepsilon} \\
\sigma_{0}^{\varepsilon}=A_{I}(x / \varepsilon) e\left(u_{0}^{\varepsilon}\right) & \text { in } I_{\varepsilon} \\
u_{0}^{\varepsilon}=0 & \text { on } \partial \Omega\end{cases} \\
\begin{cases}\operatorname{div} \xi^{\varepsilon}-\frac{1}{p} g_{0}=0 & \text { in } \Omega \\
\xi^{\varepsilon}=C_{M, p}(x / \varepsilon) e\left(\theta^{\varepsilon}\right) & \text { in } M_{\varepsilon} \\
\xi^{\varepsilon} \nu_{\varepsilon}=\left(\bar{\lambda}_{p}\left[\theta^{\varepsilon}\right]_{\varepsilon} \cdot \nu_{\varepsilon}+2 \bar{\mu}_{p}\left[\theta^{\varepsilon}\right]_{\varepsilon} \otimes_{S} \nu_{\varepsilon}\right) \nu_{\varepsilon} & \text { on } S_{\varepsilon} \\
\xi^{\varepsilon}=C_{I, p}(x / \varepsilon) e\left(\theta^{\varepsilon}\right) & \text { in } I_{\varepsilon}\end{cases}
\end{gathered}
$$

where $I_{\varepsilon}=\bigcup_{j \in J_{\varepsilon}} \varepsilon(j+I), M_{\varepsilon}=\Omega \backslash \bar{I}_{\varepsilon}, S_{\varepsilon}=\partial I_{\varepsilon}, \nu_{\varepsilon}$ is a unit normal vector to $S_{\varepsilon}$ and $[\cdot]_{\varepsilon}$ is the jump across $S_{\varepsilon}$. As previously, the interphase occupying the thin layers $B_{h \epsilon}$ is replaced by a mechanical constraint of same type along the interface $S_{\epsilon}$ the layers shrink to.

Then letting $\varepsilon$ tend to zero and proceeding as in Sanchez-Palencia [10], and Attouch-Murat [22], it can be shown that $\left(u_{0}^{\varepsilon}, \sigma_{0}^{\varepsilon}\right),\left(\theta^{\varepsilon}, \xi^{\varepsilon}\right)$ will tend to the solution of a standard homogeneous elasticity problem with effective coefficients of elasticity given by (14) and (15). Thus the limiting processes $\epsilon \rightarrow 0$ and $s^{\prime} \rightarrow 0$ do commute when $\bar{\mu}_{e} \neq 0$ and $\bar{\mu}_{v} \neq 0$. Actually this case which involves free rigid motions in the inclusions is delicate. Nevertheless, by arguing as in [23] and duly taking the connectivity of the domain $M_{\varepsilon}$ occupied by the matrix into account, it is possible to show [20] that the effective coefficients 
are also given by (14) and (15) when $\varepsilon$ and $s^{\prime}$ go quite independently to zero under the sole conditions (5) and

$$
\lim _{s \rightarrow 0} \epsilon^{2} / \mu_{e}=\lim _{s \rightarrow 0} \epsilon^{2} / h=0
$$

which means that the Lamé coefficient $\mu_{e}$ and the thickness of the interphase are not too small.

Finally, to determine the structure of the effective constitutive equations of the real media, it is fundamental to note that $C^{\mathrm{eff}}(p)$ is not equal to $\frac{1}{p} A^{\mathrm{eff}}(p)+B^{\mathrm{eff}}$, but that we have :

$$
C^{\mathrm{eff}}(p)=\hat{K}(p)+\frac{1}{p} A^{\mathrm{eff}}+B^{\mathrm{eff}}
$$

with the tensor $K$ being defined by :

$$
K_{i j k l}(t):=\frac{1}{|Y|} \int_{Y}\left(\tau^{k l}\right)_{i j}(y, t) \mathrm{d} y
$$

where $\left(w^{k l}, \tau^{k l}\right)$ is the unique solution, up to a constant for $w^{k l}$, to the evolution problem :

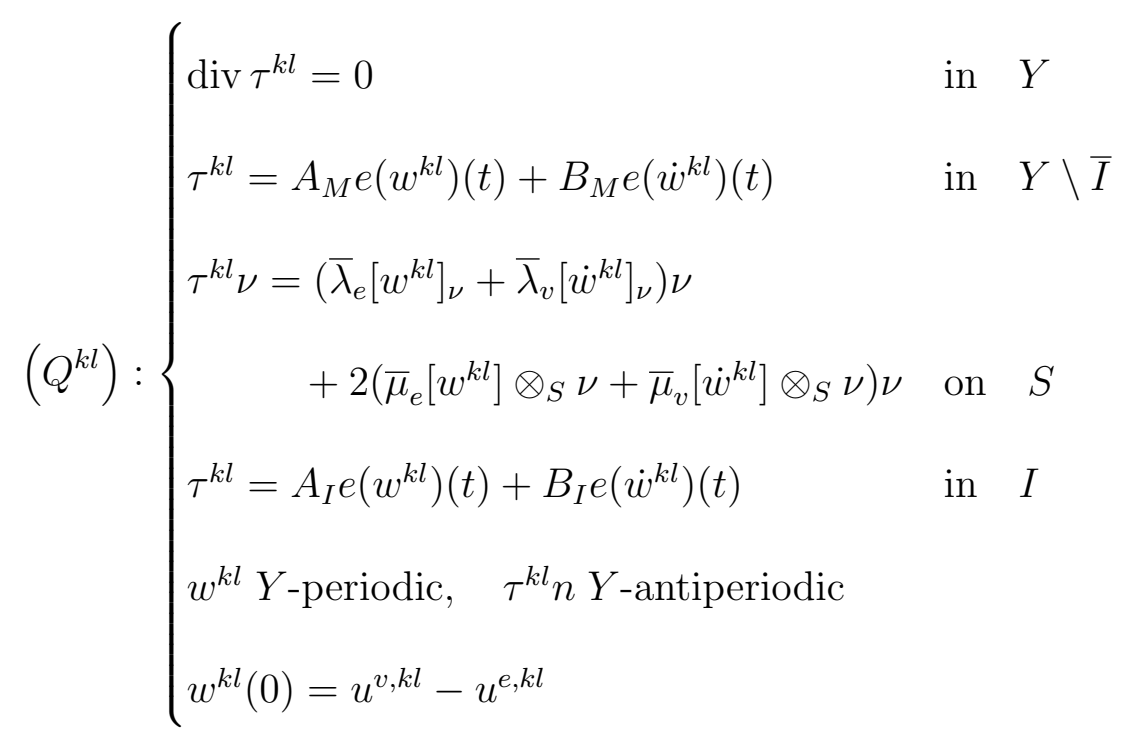

when some coefficients $\bar{\lambda}_{e}, \bar{\mu}_{e}, \bar{\lambda}_{v}, \bar{\mu}_{v}$ are not finite, the term involved in the 
boundary conditions on $S$ for $\left(P^{v, k l}\right),\left(P^{e, k l}\right)$ and $\left(Q^{k l}\right)$ disappears and is replaced by the condition $[\cdot]_{\nu}=0$ when $\left\{\bar{\lambda}_{e}, \bar{\lambda}_{v}\right\} \ni \infty$ or $[\cdot]=0$ when $\left\{\bar{\mu}_{v}, \bar{\mu}_{e}\right\} \ni \infty$. This corresponds to the perfect bonding case, i.e. continuity of displacement and of the stress vector across the interface.

Hence (19) implies that the effective behavior of the composite is described by displacement and stress fields $\left(u^{0}, \sigma^{0}\right)$ satisfying :

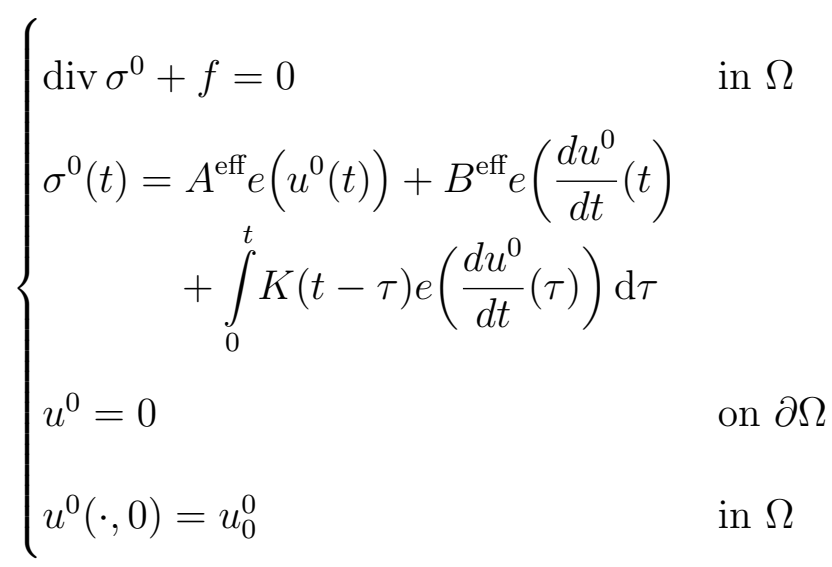

The fact that $C^{\text {eff }}(p)$ differs from $\frac{1}{p} A^{\text {eff }}(p)+B^{\text {eff }}$ (see (19)) is the source of the memory term. As in [12] it can be shown that $K(t)$ possesses the classical symmetries and exponentially decreases when $t$ goes to $+\infty$. The homogeneous material is no longer of Kelvin-Voigt type but rather a material with fading memory. Actually, this fading memory effect is due to the incompatibility of the relaxation times of the three phases and has been evidenced in many works. Note that if the initial state $u_{0}^{s}$ does not satisfy (4), an additional term involving $u_{0}^{s}$ appears in the homogenized stress $\sigma^{0}$. 


\section{$3 \quad$ Numerical Experiments}

The homogenized terms $A^{\text {eff }}$ and $B^{\text {eff }}$ have been computed in [24], so we focused on the study of the influence of various parameters on the sole kernel associated with the memory term. To reduce the computational work, we make the assumption that the inclusions are long parallel fibers and that the material properties are independent with respect to the variable parallel to the fibers. Hence the frame work of plain strain may be used and the kernel $K$ reduces to a $3 \times 3$ symmetric matrix $\bar{K}$ connecting the components $\left(E_{11}, E_{22}, E_{12}\right)$ of the strain tensor to the components $\left(\Sigma_{11}, \Sigma_{22}, \Sigma_{12}\right)$ of the stress tensor, where 1 and 2 refer to two orthogonal directions normal to the fiber direction. The fiber cross-sections are assumed to be circular and centered in a unit square, then we have $\bar{K}_{22}=\bar{K}_{11}$ and $\bar{K}_{23}=\bar{K}_{13}$.

A finite element code, namely CAST3M $\mathrm{R}$ [25], was used for the computations. A typical mesh used in the numerical experiments in case of a $50 \%$ volume fraction of inclusions is shown in Figure 4. The amplitude of the time evolution study is $T=10^{-3} s$ and an implicit method with a time step $\delta t=10^{-5} s$ is used. Hereafter, the superscripts inc and mat refer to the mechanical characteristics of the isotropic inclusion and isotropic matrix, respectively. First, note that all the presented curves exhibit the same trend, i.e. the influence of the memory term vanishes after a reasonable time. In what follows, for any decreasing function $G$ of the time, the "characteristic decay time" of $G$ means the time $t_{c}$ such that $G\left(t_{c}\right)$ is equal to $10 \%$ of its initial value $G(0)$. 
3.1 Influence of material and geometrical parameters in case of perfect bonding

First, Figures 5 and 6 show the influence of the contrast of the elastic properties of the inclusions and the matrix in case of perfect bonding. We set the volume fraction of inclusions at $50 \%$ and the material properties as indicated in Table 1 . The perfect bonding of the inclusion to the matrix is numerically obtained by setting very high values for the mechanical interface parameters, namely $\left\{\bar{\lambda}_{v}, \bar{\mu}_{v}\right\}$ and $\left\{\bar{\lambda}_{e}, \bar{\mu}_{e}\right\}$. For this parametric study, the ratio $R_{L_{c}}=\frac{\lambda_{e}^{i n c}}{\lambda_{e}^{m a t}}=\frac{\mu_{e}^{i n c}}{\mu_{e}^{m a t}}$ between the Lamé coefficients of the inclusion and of the matrix ranges from $2.10^{-2}$ to 4 . Of course, when $R_{L_{c}}=1$, i.e. matrix and inclusion are similar, the memory term $\bar{K}$ vanishes. When $R_{L_{c}}$ increases from 0.02 to 1., i.e. soft inclusion cases, all of the initial values of $\bar{K}$ decrease and tend to vanish for $R_{L_{c}}=1$. When $R_{L_{c}}$ increases from 1. to 4., i.e. stiff inclusion cases, the initial value of all components of $\bar{K}$ increases. We also note that "characteristic decay time" of each component of $\bar{K}$ is lower for stiff inclusions than for soft inclusions for a given volume fraction of inclusions.

The influence of the volume fraction of inclusions on the memory term is then presented in the case of a stiff inclusion $\left(R_{L_{c}}=0.1\right)$ with material constants as in Table 2 and of a soft inclusion $\left(R_{L_{c}}=10\right)$ with material constants as in Table 3. Here the volume fraction of inclusions ranges from $10 \%$ to $70 \%$. For clarity, only studies with coefficients $\bar{K}_{11}$ and $\bar{K}_{12}$ are presented respectively in Figures 7 and 8. In case of a stiff inclusion, Figures 7(a) and 8(a) show that the initial value of the memory term and its decay increase with the volume fraction of inclusions. In case of a soft inclusion, Figures 7(b) and 8(b) show that the lowest initial value is also associated with the lowest value of the 
volume fraction of inclusions used here, namely 10\%. This could be linked to the fact that for very low volume fractions the inclusion has no influence since the memory term tends to zero [13]. Figure 8(b) also indicates that this initial value peaks around $40 \%$ of volume fraction of inclusions. As for a stiff inclusion, the initial value first increases with the increase in the volume fraction of inclusions and secondly for large volume fractions, namely $V_{\text {frac }} \geq 40 \%$, the initial value still decreases due to the influence of the inclusion stiffness. The existence of this maximum highlights that for high volume fractions of inclusions the memory term is governed by the constitutive behavior of the inclusion and for low volume fractions of inclusions the memory term tends to vanish. In the case of a large volume fractions of soft inclusion, Figures 7 (b) and 8(b) illustrate a decrease in the "characteristic decay time" of $\bar{K}_{11}$ and $\bar{K}_{12}$, respectively, with an increase in the volume fraction of inclusions. Figures 7 and 8 also show that the composite with a stiff inclusion exhibits the lowest "characteristic decay time" of $\bar{K}_{i j}$. The results obtained in the two previous parametric studies are in total agreement with the results proposed by Cherraf-Schweyer et al. [13].

A parametric study highlighting the influence of the interface material characteristics on the memory term is now presented. Hereafter, we set the volume fraction of inclusions at $50 \%$ and the material properties of the matrix and the inclusions are defined in Table 1 . Only numerical experiments on $\bar{K}_{11}$ are presented for the sake of clarity. 


\subsection{Influence of the elastic properties of the interface}

First the influence of the elastic constitutive coefficients of the interface is studied for a stiff or soft inclusion for the two values of the viscosity constitutive coefficients of the interface given in Table 4 and respectively on Figures 9 and 10. In theses figures, a set of curves are presented for $R_{k}=\frac{\bar{\lambda}_{e}}{\bar{\lambda}_{e_{0}}}$ ranging from 1 to $10^{-3}$. In the case of high values of the viscosity constitutive coefficients of the interface, Figure 9(a) clearly shows an increase in the "characteristic decay time" of $\bar{K}_{11}$ with the decrease in $R_{k}$. Figure 9 (a) also exhibits that the decay of $\bar{K}_{11}$ always exist as predicted by theory even if it might take much longer time. This phenomena is also observed in [13]. Figure $9(\mathrm{~b})$ is a re-scale of Figure 9(a) to point out what is happening in case of $R_{k} \in[0.5,1]$. This increase is much greater with the stiff inclusion than with the soft inclusion. This highlights an influence of the elastic coefficient of the interface on the decay of $\bar{K}_{11}$ accentuated by the stiffness of the inclusion even for high values of $\left\{\bar{\lambda}_{v}, \bar{\mu}_{v}\right\}$. In the case of low values of the viscosity constitutive coefficients of the interface, Figure 10 indicates a decrease in the initial value of $\bar{K}_{11}$ with a decrease in $R_{k}$. In this case, there is no significant effect of $R_{k}$ on the "characteristic decay time" of $\bar{K}_{11}$.

\subsection{Influence of the viscous properties of the interface}

Now the influence of the viscosity constitutive coefficients of the interface is studied. We keep the same geometrical and material parameters as before (Table 2) for the matrix and inclusion. In Figures 11 and 12, sets of curve values are presented for $R_{v i s}=\frac{\bar{\lambda}_{v}}{\bar{\lambda}_{v 0}}$ ranging from 1.0 to $10^{-3}$. In Figure 11, we 
observe that for high values of $\overline{\lambda_{e}}$ and $\overline{\mu_{e}}$ (i.e. very stiff elastic interface), there is no influence of $\overline{\lambda_{v}}, \overline{\mu_{v}}$ on the behavior of $\bar{K}_{11}$. In fact, high elastic stiffness of the interface does not favor the relative displacement along the interface, so this is very close to a quasi-perfect bonding case then the viscosity coefficients have no influence on $\bar{K}_{11}$. On the contrary, in Figure 12 , we observe a marked influence of $\{\bar{\lambda}, \bar{\mu}\}_{v}$ on the memory term $\bar{K}_{11}$ which corresponds to a weak elastic stiffness of the interface. In the case of a stiff inclusion in Figure 12(a), an increase in the "characteristic decay time" of $\bar{K}_{11}$ is observed. This time increases with $R_{v i s}$. In the case of a soft inclusion, as shown in Figure 12(b), a less marked influence is noticed as in the previous parametric study. These results are in agreement with the curves presented in Figure 9.

\section{Conclusion}

The numerical experiments confirmed the evanescent character of the memory term and illustrate the high dependency on the geometrical and material parameters. For quasi-perfect bonding, we notice that the influence of the volume fraction of inclusions may change according to the contrast in the elastic properties of the matrix and inclusions. For stiff inclusion, the increase of initial values and "characteristic decay time" of all components of $\bar{K}$ are directly linked to the increase in the inclusion volume fraction. It is also shown that low values of the elastic parameters of the interface significantly affect the variations with respect to the time of $\bar{K}$, especially in the case of a stiff inclusion. The parametric study also showed that the influence of the interface parameter on the response of the memory term is greater when the elastic properties of the interface are weak. This result is in accordance with those of 
[26], thus highlighting the importance of the weakness of the elastic properties in the damping of the composite plate. Further studies should be carried out to assess the influence of a viscous interface in a three-phase composite on soundproofing.

\section{Références}

[1] H. Duan, X. Yi, Z. Huang, J. Wang, A unified scheme for prediction of effective moduli of multiphase composites with interface effects : Part ii, Application and scaling laws, Mechanics of Materials 39 (1) (2007) $94-103$.

[2] H. Shen, P. Schiavone, C. Ru, A. Mioduchowski, Stress analysis of an elliptic inclusion with imperfect interface in plane elasticity, Journal of Elasticity 62 (2001) 25-46.

[3] P. Mallick, Fibre-reinforced Composites : Materials, Manufacturing, and Design, CRC Press, Taylor, Francis Group, 2007.

[4] S. A. Meftah, E. M. Daya, A. Tounsi, Finite element modelling of sandwich box column with viscoelastic layer for passive vibrations control under seismic loading, Thin-Walled Structures 51 (0) (2012) 174 - 185. doi:http://dx.doi. $\operatorname{org} / 10.1016 / j \cdot t w s .2011 .10 .015$.

[5] F. Boumediene, J.-M. Cadou, L. Duigou, E. Daya, A reduction model for eigensolutions of damped viscoelastic sandwich structures, Mechanics Research Communications 57 (0) (2014) 74 - 81. doi:http://dx.doi.org/10.1016/j. mechrescom.2014.03.001.

[6] M. Gandel'sman, S. Tsygankov, A. Gol'dman, V. Budtov, Prediction of the viscoelastic properties of granular composites. Dynamic characteristics of abs 
plastics., Mechanics of Composite Materials 18 (1) (1982) 105-109. doi:10. 1007/BF00605103.

[7] P. Krawczak, A. Bergere, Caractérisation et propriétés d'usage des composites〜 : Liaison renfort/matrice \& Modélisation de l'interface, Editions T.I., 2006.

[8] P. Mateille, L. Daridon, O. Arnould, A.Fanget, E. Lapebie, Mise en ouvre expérimentale d'impacts basse vitesse sur un matériau énergétique, in : CFM09 Marseille, 2009.

[9] P. Mateille, S. Coussy, L. Daridon, O. Arnould, A. Fanget, E. Lapebie, Development of an experimental device for low velocity impacts on energetic material, in : Dymat 2009 - 9th International conference on the mechanical and physical behaviour of material under dynamic loading, Vol. 1, 2009, pp. $367-372$.

[10] E. Sanchez-Palencia, Non-Homogenous Media and Vibration Theory, Lecture Notes in Physics, Springer-Verlag, 1980.

[11] G. Francfort, D. Leguillon, P. Suquet, Homogénéisation de milieux viscoélastiques linéaires de Kelvin-Voigt, C.R.A.S. Paris(1) 296 (7) (1983) 287290.

[12] G. Francfort, P. Suquet., Homogenization and mechanical dissipation in thermoviscoelasticity, Archive for Rational Mechanics and Analysis 96 (3) (1986) 265-293. doi:10.1007/BF00251909.

[13] C. Cherraf-Schweyer, G. Maurice, M. Taghite, K. Taous, Periodic homogenization in viscoelasticity. Influence of micro mechanical parameters on the homogenized memory law : numerical tests, ZAMM 87 (3) (2007) 235-246.

[14] H. Gründemann, E. Sanchez-Palencia, Homogenization techniques for composite media. proceedings, udine, italy 1985. berlin etc., springer-verlag 1987. ix, 397 pp., dm 73,- . isbn 3-540-17616-0 (lecture notes in physics 272), 
ZAMM - Journal of Applied Mathematics and Mechanics / Zeitschrift für Angewandte Mathematik und Mechanik 68 (6) (1988) 212-212.

[15] L. He, C. Lim, Time-dependent interfacial sliding in fibre composites under longitudinal shear, Composites Science and Technology 61 (4) (2001) 579 584 .

[16] N. Konchakova, R. Mueller, F.-J. Barth, Damage progress in metal/fiberreinforced polymer structures with different viscoelastic interface geometries, in : 11th International Conference on the Mechanical Behavior of Materials (ICM11), Vol. 10, 2011, pp. $758-763$.

[17] Y. Othmani, L. Delannay, I. Doghri, Equivalent inclusion solution adapted to particle debonding with a non-linear cohesive law, International Journal of Solids and Structures 48 (24) (2011) 3326 - 3335.

[18] Y. Koutsawa, M. Haberman, E. Daya, M. Cherkaoui, Multiscale design of a rectangular sandwich plate with viscoelastic core and supported at extents by viscoelastic materials, International Journal of Mechanics and Materials in Design 5 (2009) 29-44.

[19] Y. Koutsawa, M. Cherkaoui, E. Daya, Multicoating inhomogeneities problem for effective viscoelastic properties of particulate composite materials, Journal of Engineering Materials and Technology, Transactions of the ASME 131 (2009) 0210121-02101211.

[20] C. Licht, S. Orankitjaroen, Interface effects on the effective behavior of some Kelvin-Voigt viscoelastic heterogeneous bodies, Asymptotic Analysis 90 (3-4).

[21] C. Licht, G. Michaille, A modelling of elastic adhesive bonded joints, Advances in Mathematical Sciences and Applications 7 (1997) 711-740.

[22] H. Attouch, F. Murat, Homogenization of fissured elastic materials, Publication AVAMAC 1 (1985) 85-103. 
[23] C. Licht, S. Orankitjaroen, A simplified 2-dimensional model for some elastic masonries, in : International Conference in Mathematics and Apllications ICMA-MU 2011, 17-19 December 2011.

[24] F. Léné, Contribution à l'étude des matériaux composites et de leur endommagement, thèse d' Etat, Université Pierre et Marie Curie - Paris VI.

[25] T. Charras, Notice utilisateur de cast3m, cea. 1.

[26] M. Bilasse, L. Azrar, E. Daya, Complex modes based numerical analysis of viscoelastic sandwich plates vibrations, Computers and Structures 89 (7-8) (2011) $539-555$. 


\section{Liste des tableaux}

$1 \quad$ Material parameters used in the case of perfect bonding 22

$2 \quad$ Material parameters used for stiff inclusions 22

$3 \quad$ Material parameters used for soft inclusions 23

$4 \quad$ Material parameters used for studying the influence of the interface elastic parameter

5 Material parameters used for studying the influence of the interface viscosity parameter 
Tables

\begin{tabular}{|c|c|c|c|c|c|}
\hline & Value & Unit & & Value & Unit \\
\hline$\lambda_{e}^{\mathrm{inc}}$ & 40 & $\mathrm{MPa}$ & $\lambda_{v}^{\mathrm{inc}}$ & 0.4 & $\mathrm{MPas}^{-1}$ \\
\hline$\lambda_{e}^{\mathrm{mat}}$ & 4000 & $\mathrm{MPa}$ & $\lambda_{v}^{\text {mat }}$ & 0.4 & $\mathrm{MPas}^{-1}$ \\
\hline $\bar{\lambda}_{e}$ & $510^{10}$ & $\mathrm{Nm}^{-3} \mathrm{~s}^{-1}$ & $\bar{\lambda}_{v}$ & $510^{9}$ & $\mathrm{Nm}^{-3} \mathrm{~s}^{-1}$ \\
\hline$\mu_{e}^{\mathrm{inc}}$ & 19 & $\mathrm{MPa}$ & $\mu_{v}^{\text {inc }}$ & 0.19 & $\mathrm{MPas}^{-1}$ \\
\hline$\mu_{e}^{\text {mat }}$ & 1900 & $\mathrm{MPa}$ & $\mu_{v}^{\text {mat }}$ & 0.19 & $\mathrm{MPas}^{-1}$ \\
\hline $\bar{\mu}_{e}$ & $510^{10}$ & $\mathrm{~m}^{-3} \mathrm{~s}^{-1}$ & $\bar{\mu}_{v}$ & $510^{9}$ & $\mathrm{~m}^{-3} \mathrm{~s}^{-1}$ \\
\hline
\end{tabular}

TABLE 1

Material parameters used in the case of perfect bonding

\begin{tabular}{lcccccc}
\hline & Value & Unit & & Value & Unit \\
\cline { 5 - 6 } & & & & & \\
$\lambda_{e}^{\text {inc }}$ & 40000 & $\mathrm{MPa}$ & $\lambda_{v}^{\text {inc }}$ & 0.4 & $\mathrm{MPas}^{-1}$ \\
$\mu_{e}^{\text {inc }}$ & 19000 & $\mathrm{MPa}$ & $\mu_{v}^{\text {inc }}$ & 0.19 & $\mathrm{MPas}^{-1}$ \\
$\lambda_{e}^{\text {mat }}$ & 4000 & $\mathrm{MPa}$ & $\lambda_{v}^{\text {mat }}$ & 0.4 & $\mathrm{MPas}^{-1}$ \\
$\mu_{e}^{\text {mat }}$ & 1900 & $\mathrm{MPa}$ & $\mu_{v}^{\text {mat }}$ & 0.19 & $\mathrm{MPas}^{-1}$ \\
& & & & & & \\
\hline
\end{tabular}

TABLE 2

Material parameters used for stiff inclusions 


\begin{tabular}{lllllll}
\hline & Value & Unit & & Value & Unit \\
\cline { 5 - 6 } & & & & & \\
$\lambda_{e}^{\text {inc }}$ & 400 & $\mathrm{MPa}$ & $\lambda_{v}^{\text {inc }}$ & 0.4 & $\mathrm{MPas}^{-1}$ \\
$\mu_{e}^{\text {inc }}$ & 190 & $\mathrm{MPa}$ & $\mu_{v}^{\text {inc }}$ & 0.19 & $\mathrm{MPas}^{-1}$ \\
$\lambda_{e}^{\text {mat }}$ & 4000 & $\mathrm{MPa}$ & $\lambda_{v}^{\text {mat }}$ & 0.4 & $\mathrm{MPas}^{-1}$ \\
$\mu_{e}^{\text {mat }}$ & 1900 & $\mathrm{MPa}$ & $\mu_{v}^{\text {mat }}$ & 0.19 & $\mathrm{MPas}^{-1}$ \\
\hline
\end{tabular}

TABLE 3

Material parameters used for soft inclusions

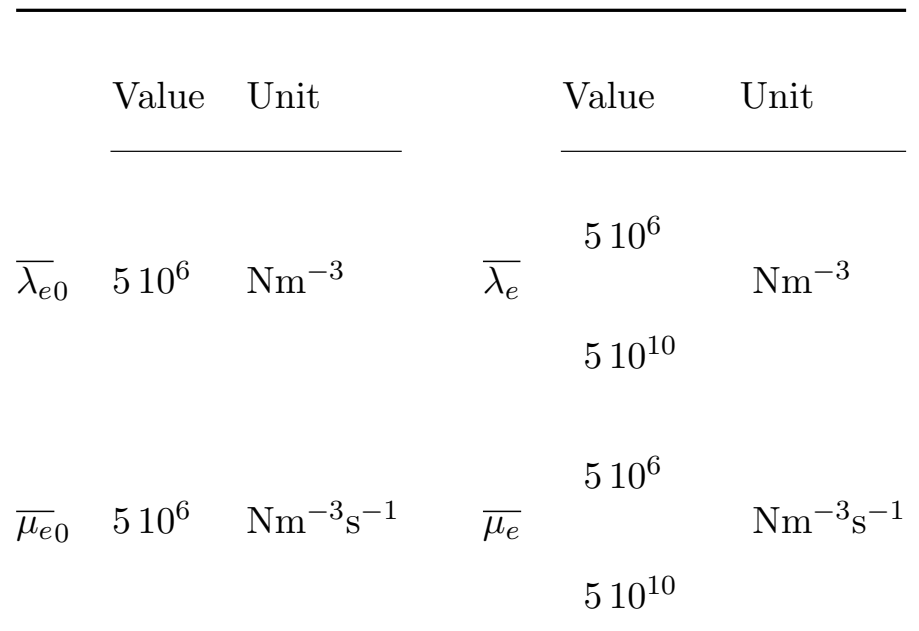

TABLE 4

Material parameters used for studying the influence of the interface elastic parameter 


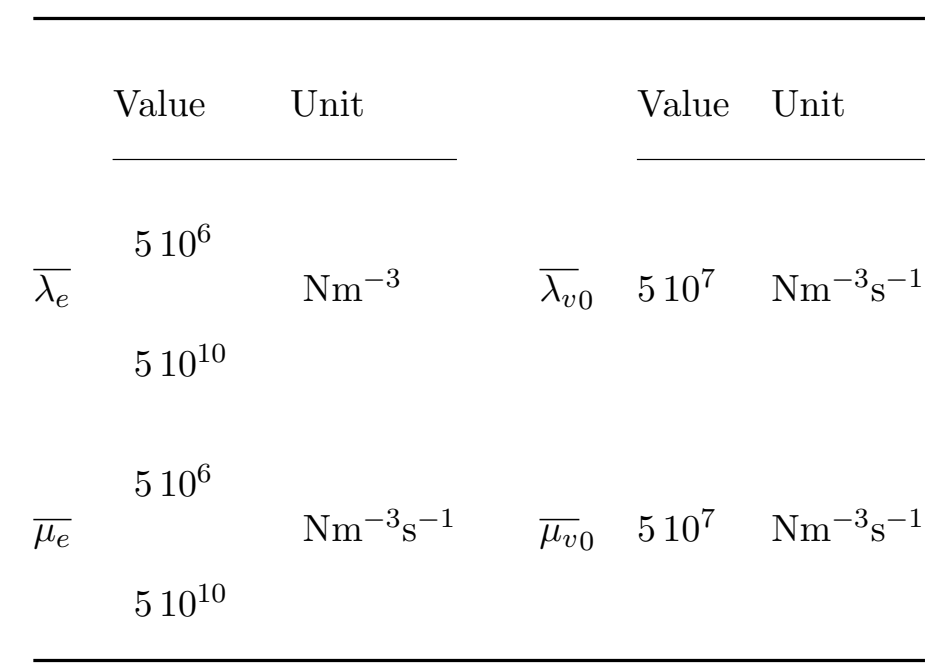

TABle 5

Material parameters used for studying the influence of the interface viscosity parameter 
Table des figures

$1 \quad$ SEM views of glass bead inclusions in a PMMA matrix without a coupling agent $\quad 26$

$2 \quad$ SEM views of glass bead inclusions in a PMMA matrix with a coupling agent

$\begin{array}{lll}3 & \text { A 2D representation of the studied geometry } & 27\end{array}$

$4 \quad$ Mesh used for the numerical experiment 27

$5 \quad$ Influence of the contrast in elastic properties on $\bar{K}_{1 i}$

$6 \quad$ Influence of the contrast in elastic properties on $\bar{K}_{33}$

$7 \quad$ Influence of the volume fraction of inclusions on $\bar{K}_{11}$

$8 \quad$ Influence of the volume fraction of inclusions on $\bar{K}_{12}$

$9 \quad$ Influence of $\bar{\lambda}_{e}, \bar{\mu}_{e}$ for high $\bar{\lambda}_{v}, \bar{\mu}_{v}$ values on $\bar{K}_{11}$

$10 \quad$ Influence of $\bar{\lambda}_{e}, \bar{\mu}_{e}$ for low $\bar{\lambda}_{v}, \bar{\mu}_{v}$ values on $\bar{K}_{11}$

11 Influence of $\bar{\lambda}_{v}, \bar{\mu}_{v}$ for high $\bar{\lambda}_{e}, \bar{\mu}_{e}$ values on $\bar{K}_{11}$

12 Influence of $\bar{\lambda}_{v}, \bar{\mu}_{v}$ for low $\bar{\lambda}_{e}, \bar{\mu}_{e}$ values on $\bar{K}_{11}$ 


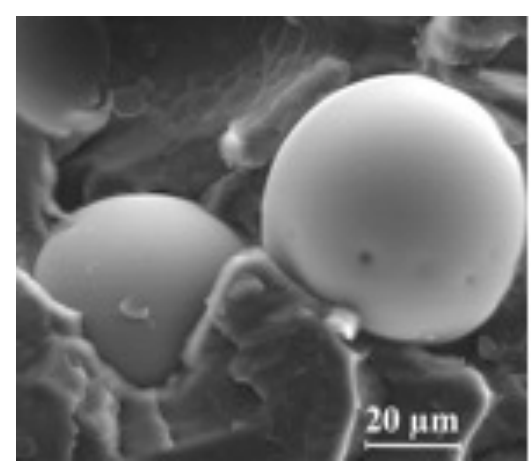

FIGURE 1. SEM views of glass bead inclusions in a PMMA matrix without a coupling agent

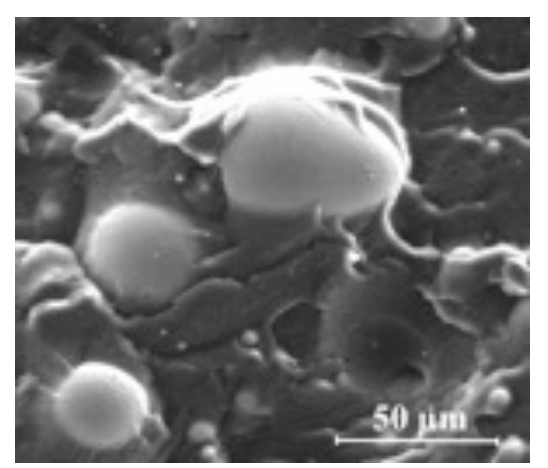

FiguRE 2. SEM views of glass bead inclusions in a PMMA matrix with a coupling agent 


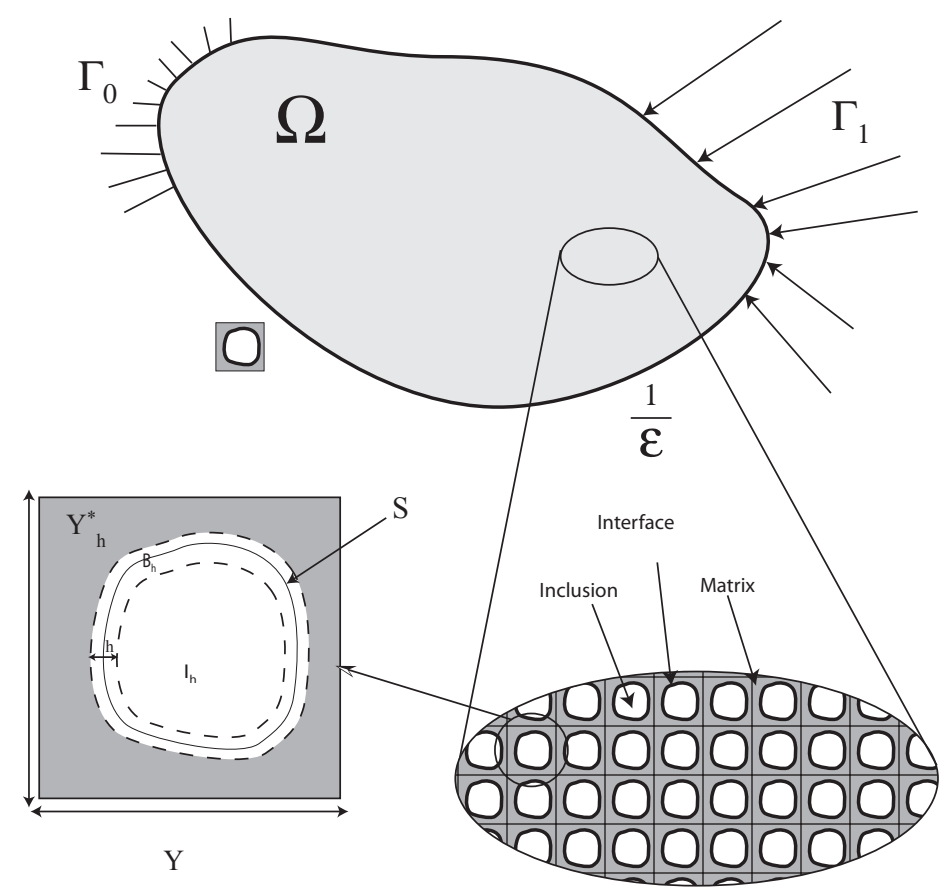

FiguRE 3. A 2D representation of the studied geometry

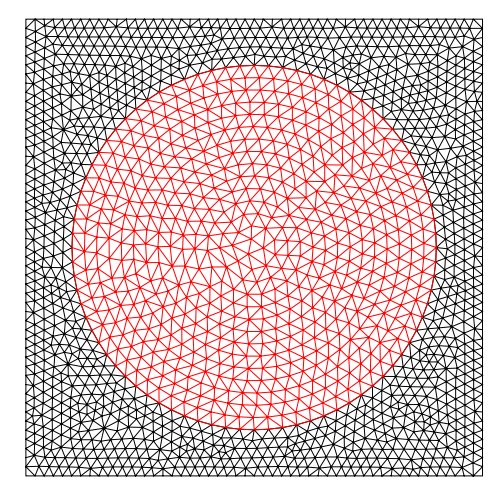

Figure 4. Mesh used for the numerical experiment 


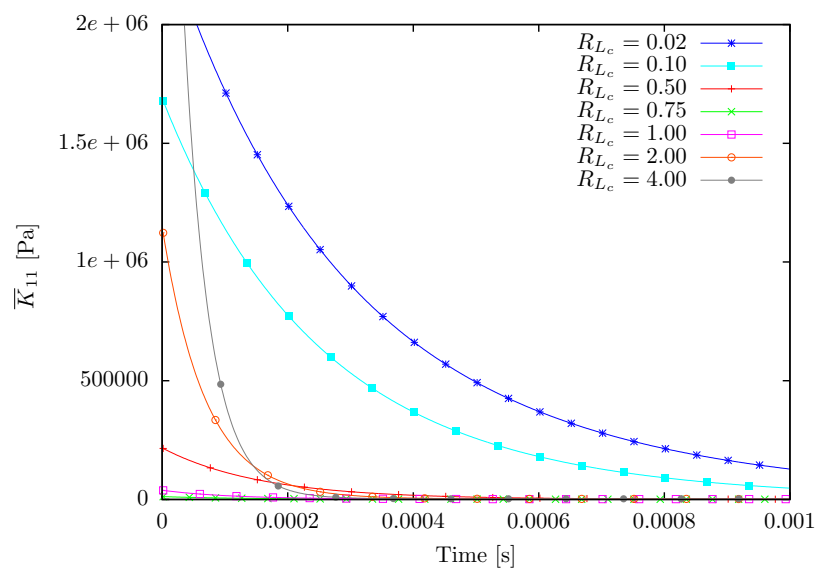

(a) on $\bar{K}_{11}$

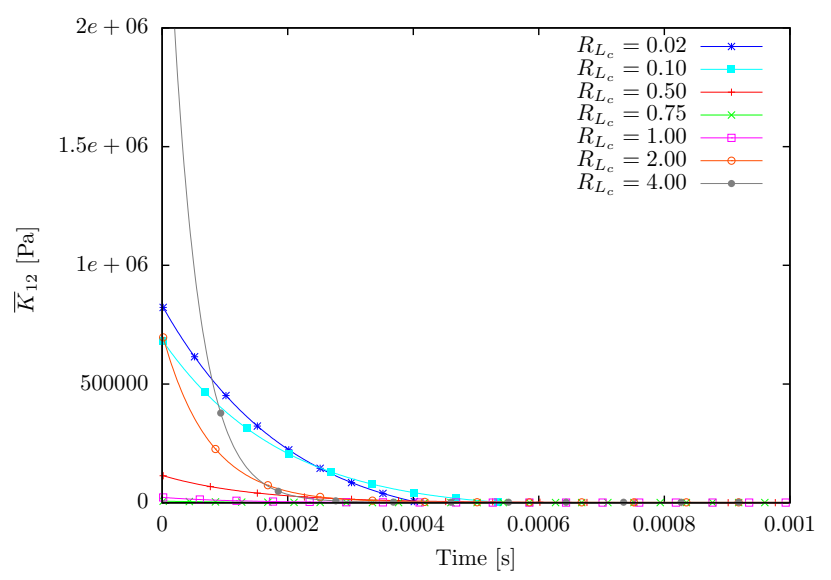

(b) on $\bar{K}_{12}$

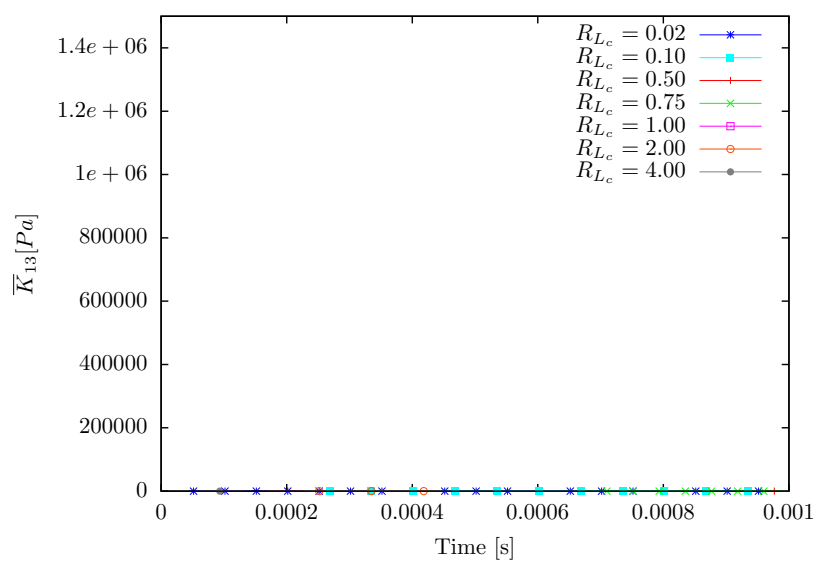

(c) on $\bar{K}_{13}$

FiguRE 5 . Influence of the contrast in elastic properties on $\bar{K}_{1 i}$ 


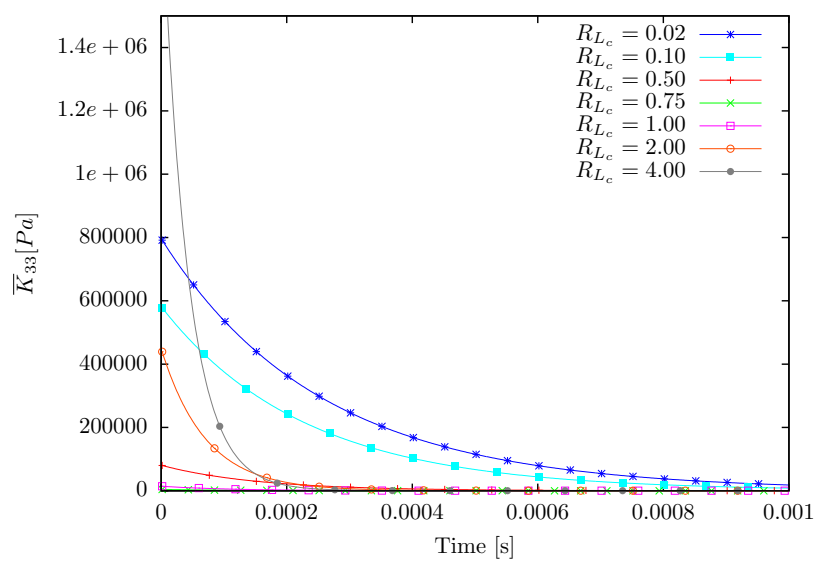

(a) on $\bar{K}_{33}$

FigURE 6 . Influence of the contrast in elastic properties on $\bar{K}_{33}$ 


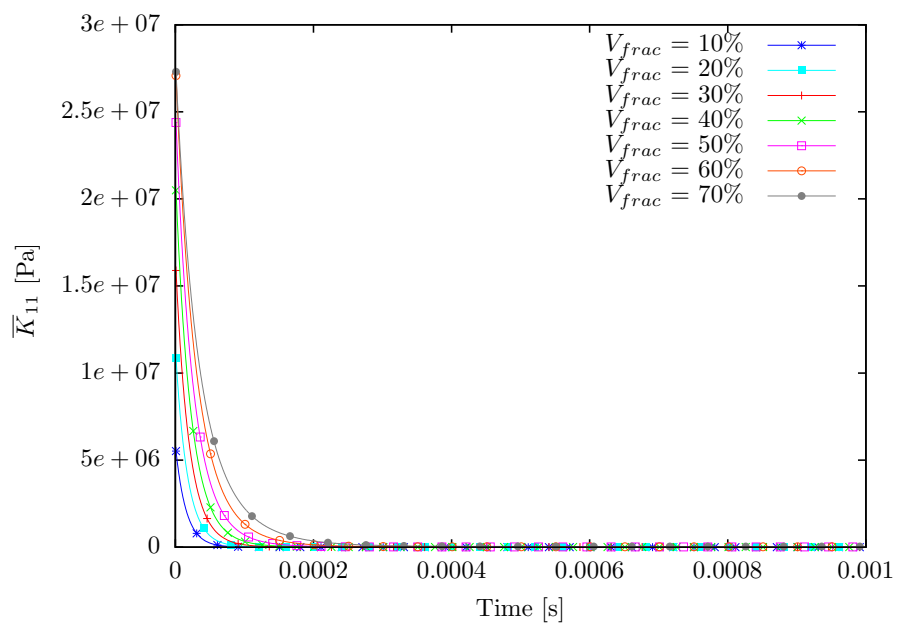

(a) Stiff inclusion

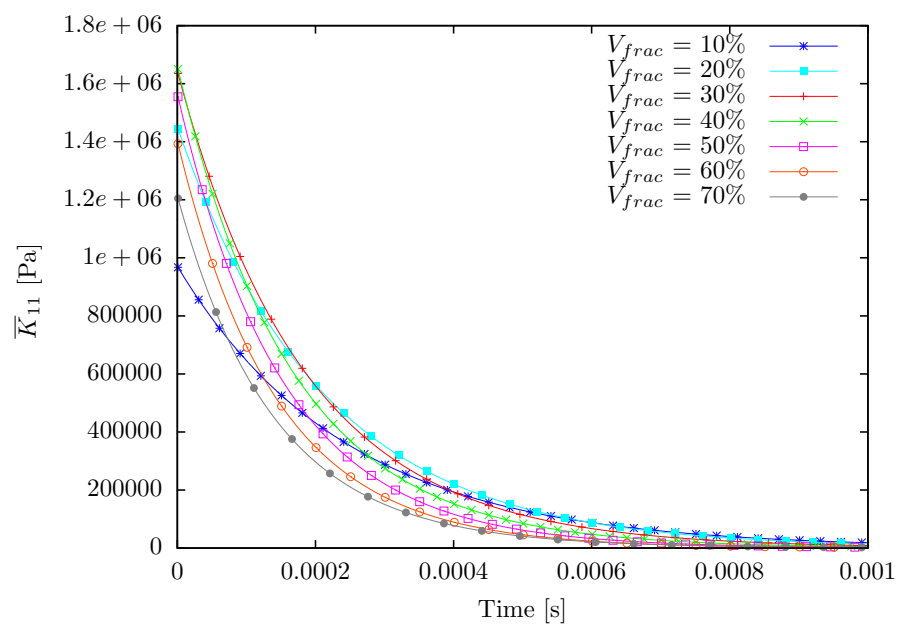

(b) Soft inclusion

FiguRE 7 . Influence of the volume fraction of inclusions on $\bar{K}_{11}$ 


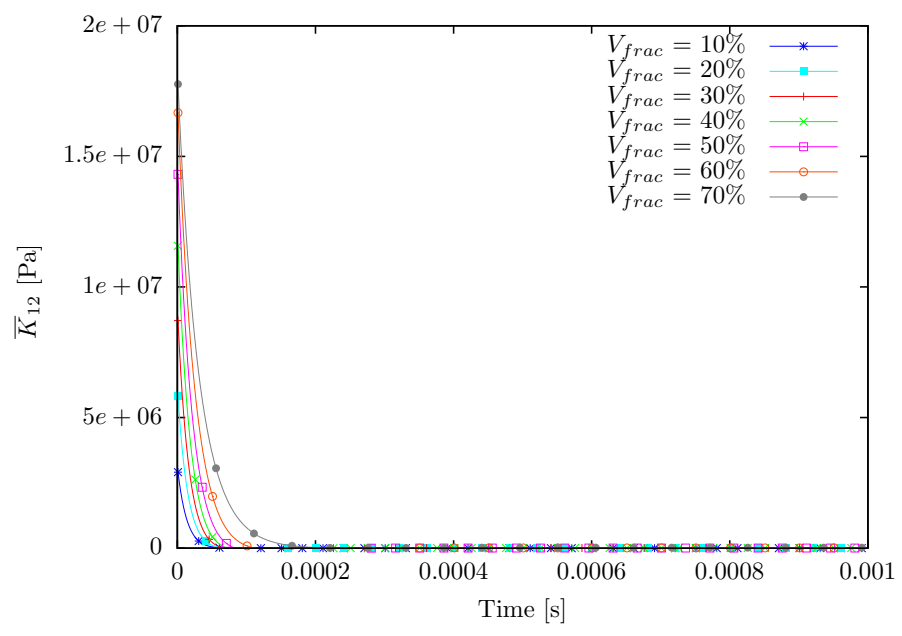

(a) Stiff inclusion

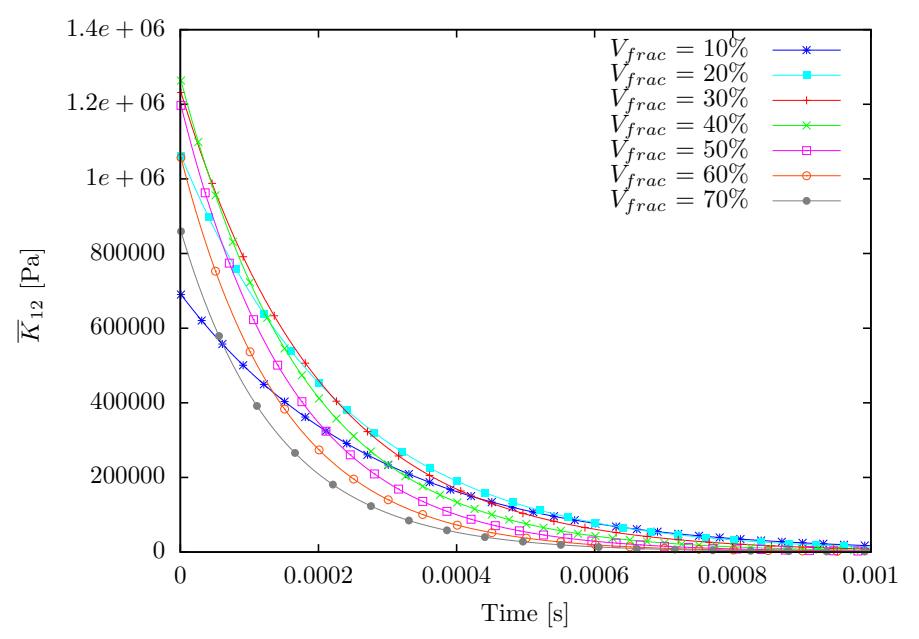

(b) Soft inclusion

Figure 8. Influence of the volume fraction of inclusions on $\bar{K}_{12}$ 


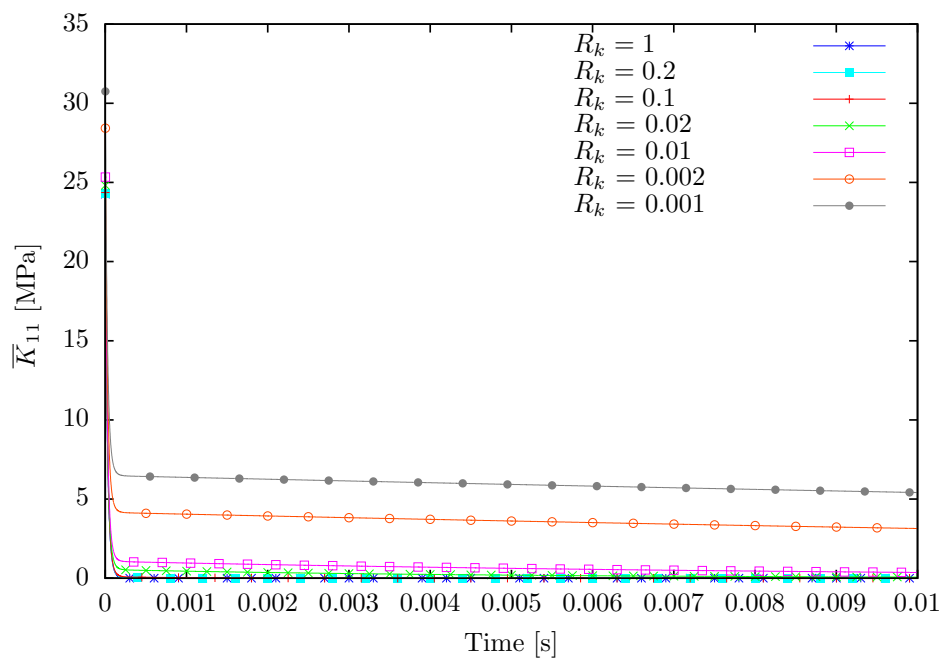

(a) Stiff inclusion

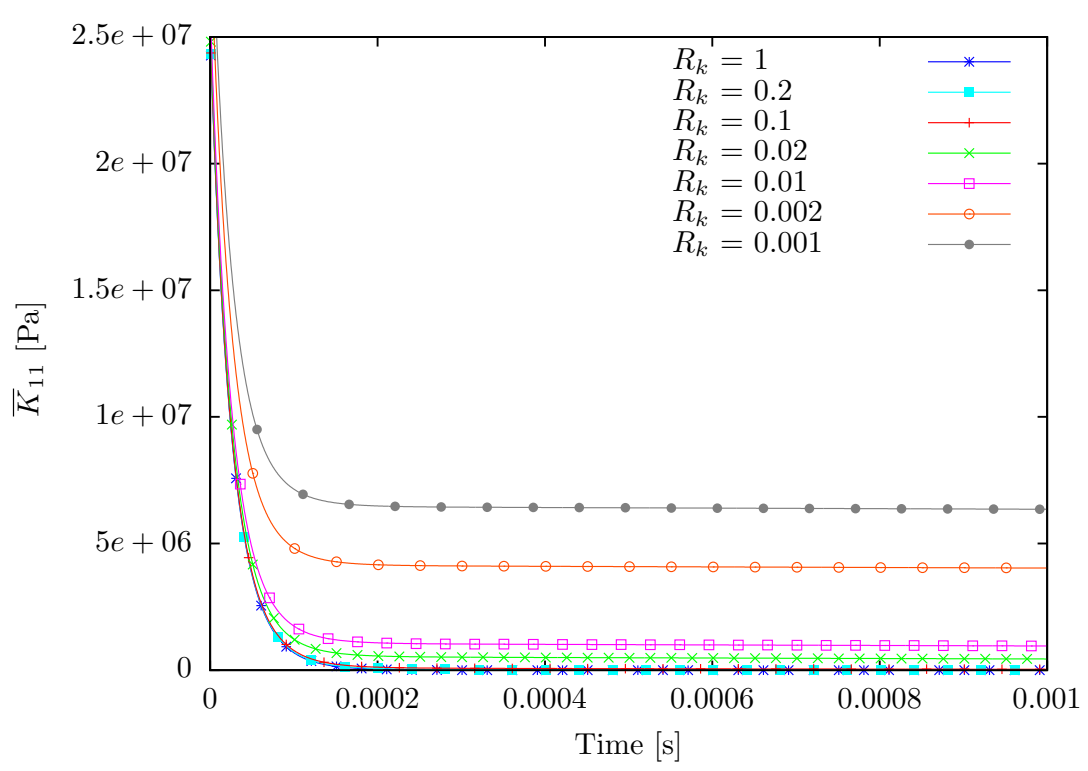

(b) Stiff inclusion

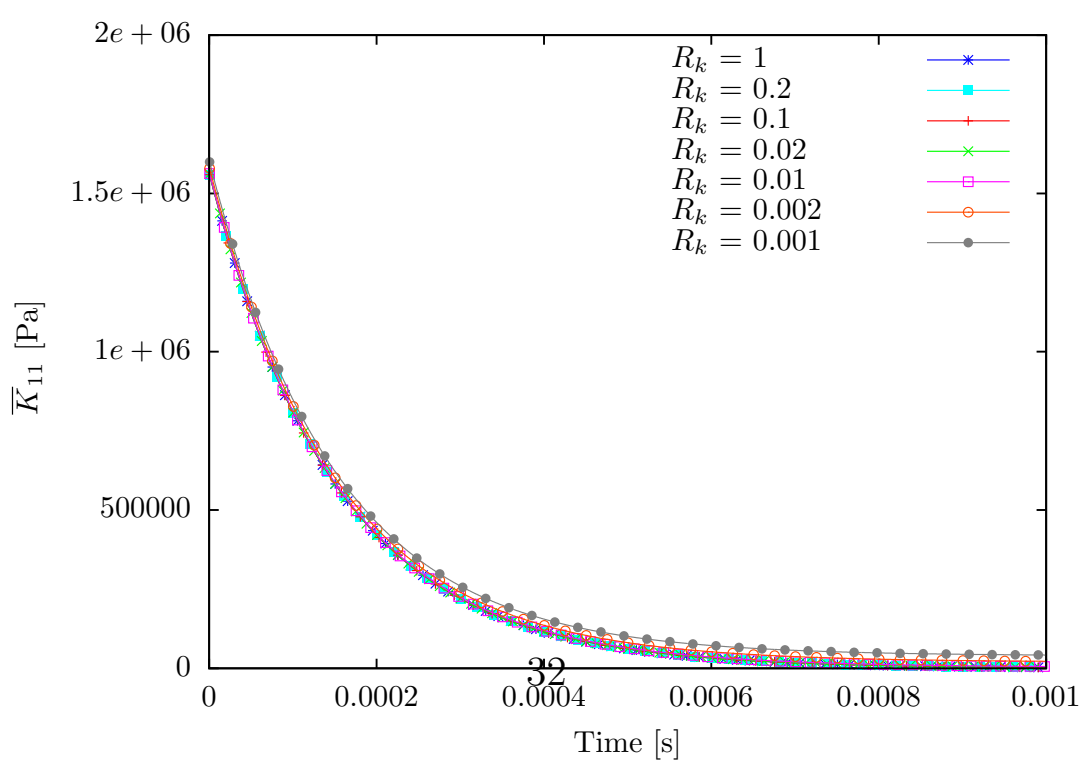

(c) Soft inclusion

FigURE 9. Influence of $\bar{\lambda}_{e}, \bar{\mu}_{e}$ for high $\bar{\lambda}_{v}, \bar{\mu}_{v}$ values on $\bar{K}_{11}$ 


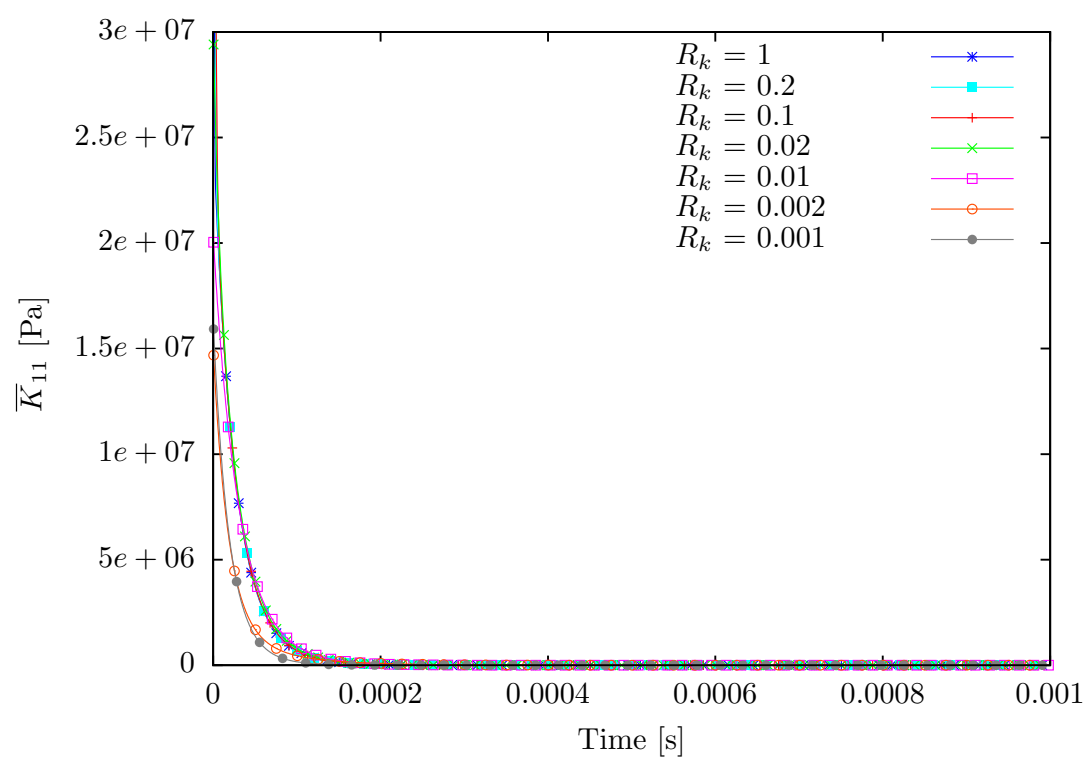

(a) Stiff inclusion

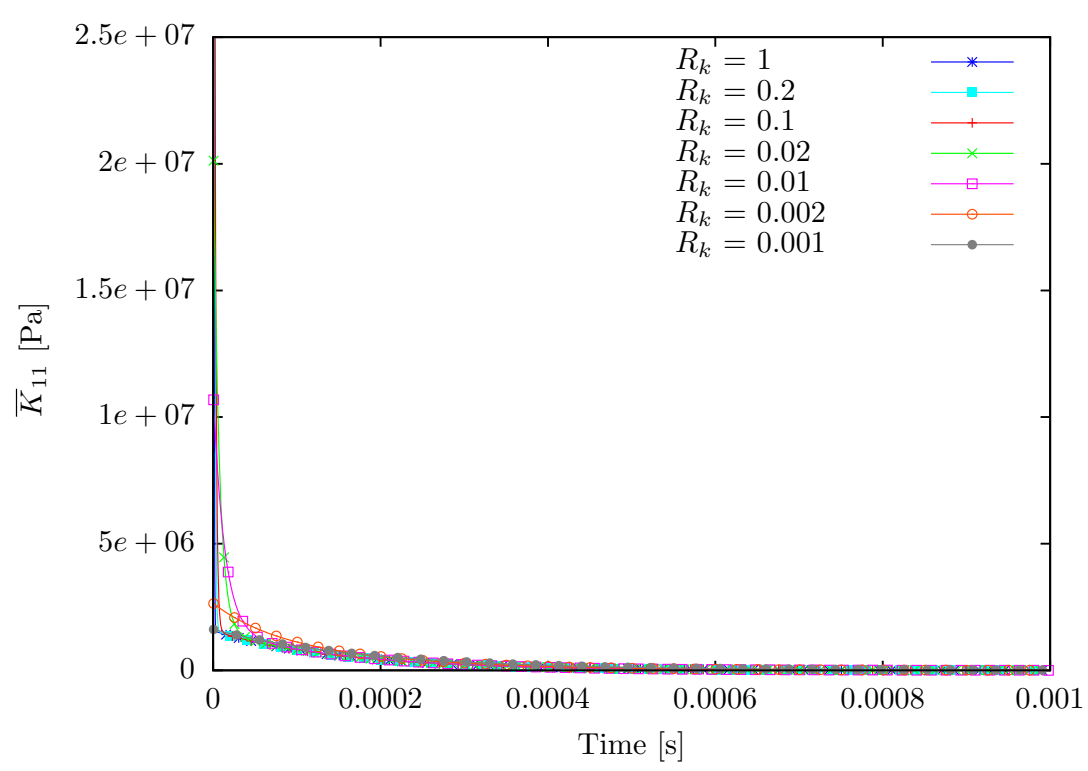

(b) Soft inclusion

Figure 10. Influence of $\bar{\lambda}_{e}, \bar{\mu}_{e}$ for low $\bar{\lambda}_{v}, \bar{\mu}_{v}$ values on $\bar{K}_{11}$ 


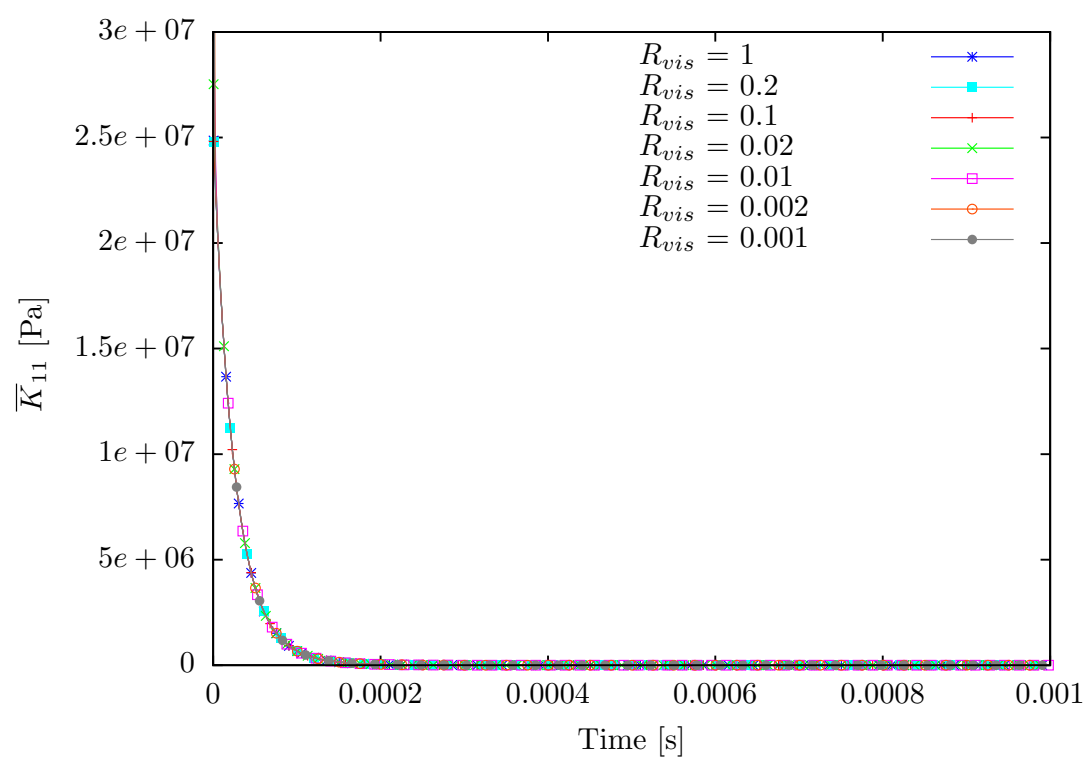

(a) Stiff inclusion

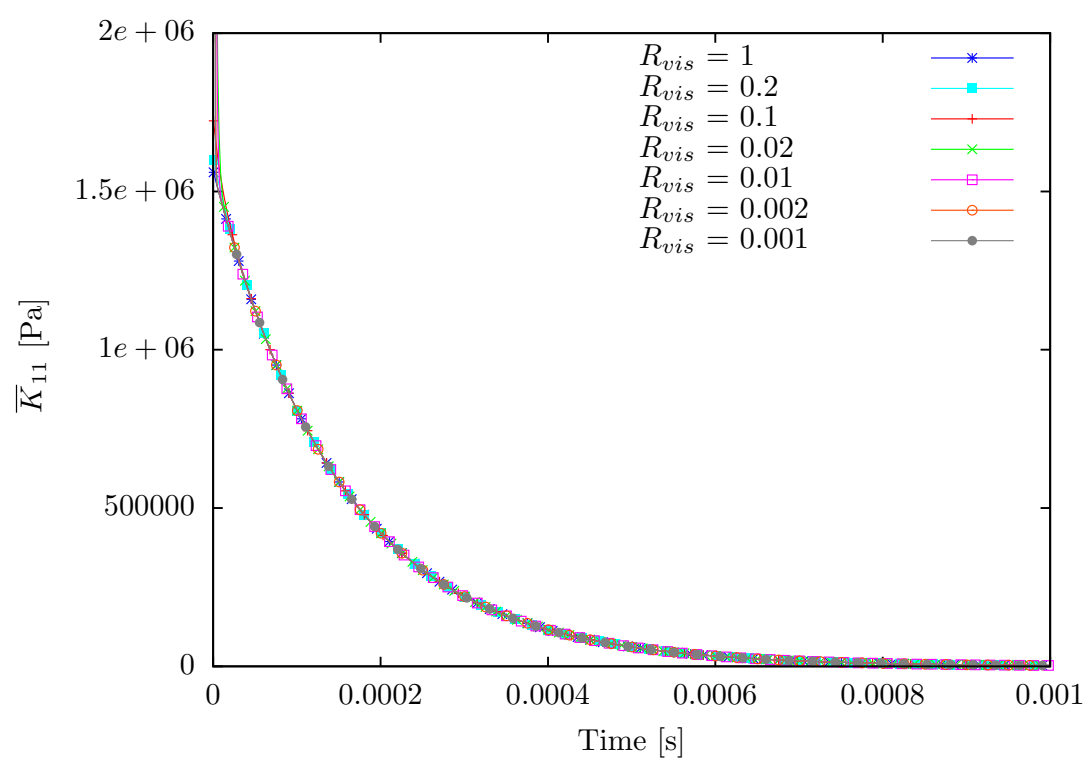

(b) Soft inclusion

Figure 11. Influence of $\bar{\lambda}_{v}, \bar{\mu}_{v}$ for high $\bar{\lambda}_{e}, \bar{\mu}_{e}$ values on $\bar{K}_{11}$ 


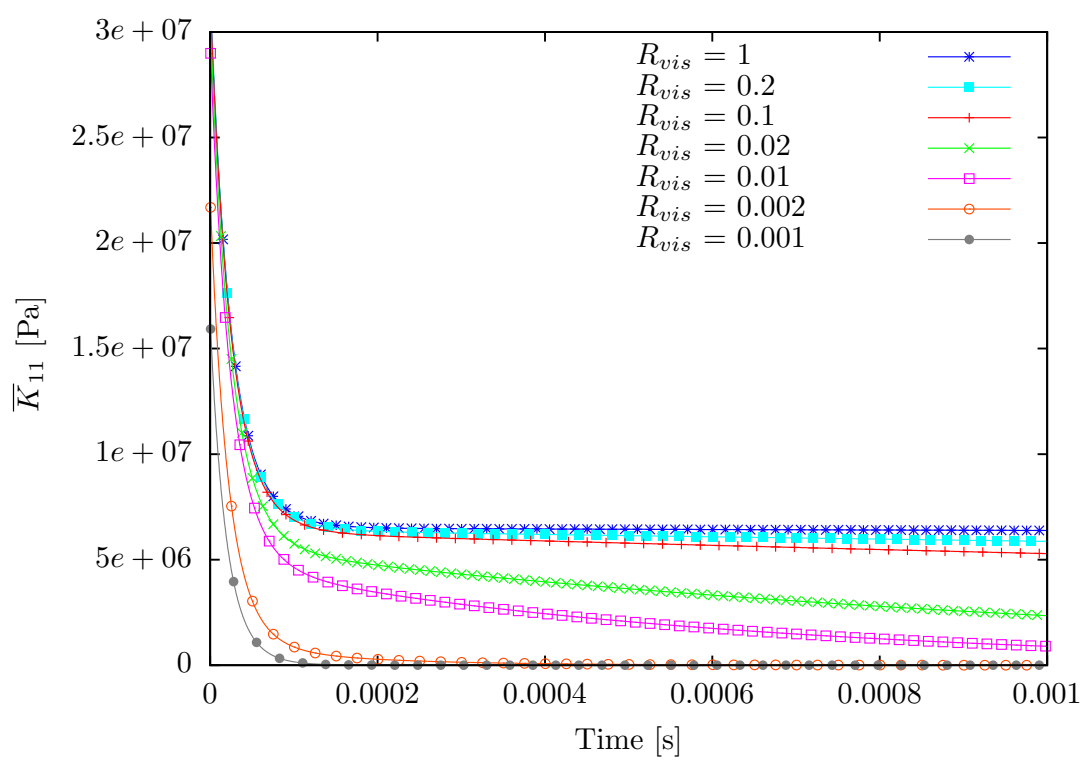

(a) Stiff inclusion

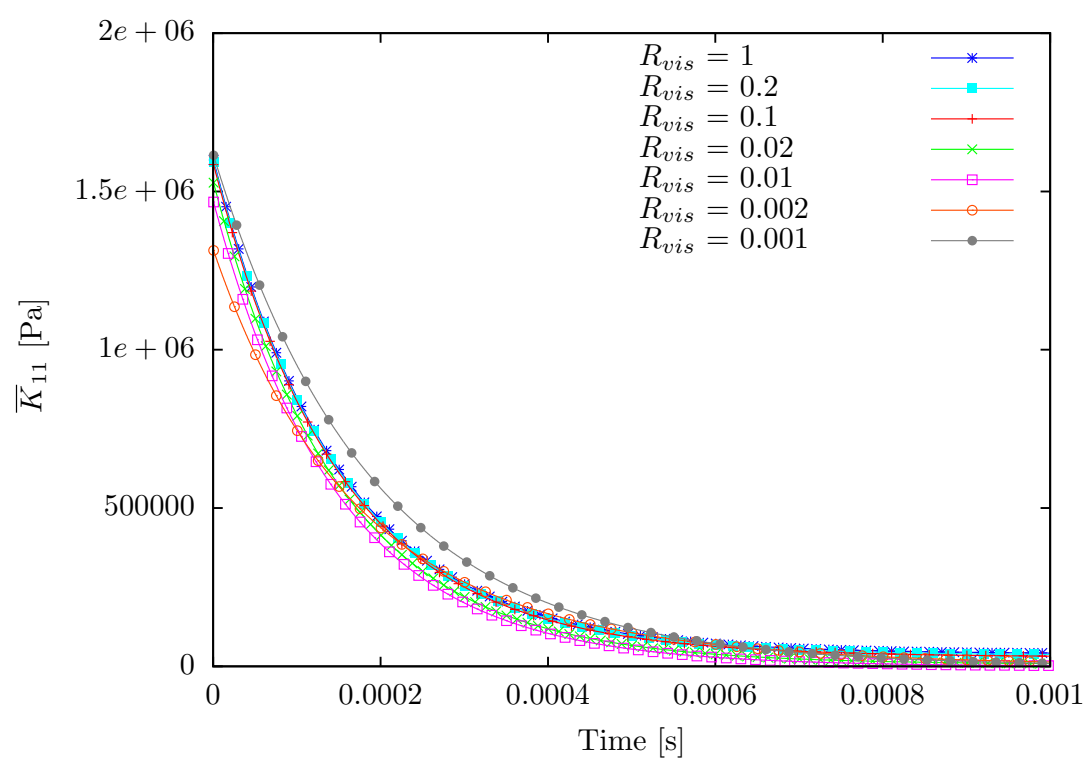

(b) Soft inclusion

Figure 12. Influence of $\bar{\lambda}_{v}, \bar{\mu}_{v}$ for low $\bar{\lambda}_{e}, \bar{\mu}_{e}$ values on $\bar{K}_{11}$ 